\title{
A Clinical Study Starting from an Encounter with a Patient
}

\author{
MASATAKA SUMIYOSHI \\ Department of Cardiology, Juntendo University Nerima Hospital, Tokyo, Japan
}

\begin{abstract}
The story of this clinical study began with a patient I encountered as a post-graduate student on night duty at Juntendo University Hospital in 1989. The patient was diagnosed as idiopathic ventricular fibrillation (IVF) with incomplete right bundle branch block and a bizarrely shaped ST segment elevation in $\mathrm{V}_{1-3}$. He had a history of sick sinus syndrome (SSS) one year earlier. Three years after that encounter, the Brugada brothers reported eight patients with IVF whose ECG findings were very similar to those of my patient, and this syndrome was later named Brugada syndrome (BrS). It is now known that there are many individuals with BrS in Japan and across Asia, and regrettably the first patient's report was not released in Japan. With the later progress in genomic research it has become apparent that a single gene mutation could be a cause of both BrS and SSS. We have begun a clinical study of the association between BrS and SSS and have published several papers about this issue. My first patient with BrS and SSS has been doing well without syncope for over 30 years.
\end{abstract}

Key words: Brugada syndrome, sinus node dysfunction, pacemaker

After graduating from the graduate medical school, I encountered a very impressive patient while I was on night duty at Juntendo University Hospital in 1989. The patient was a 42-year-old man, medical nurse, who was transferred to our hospital after experiencing several episodes of syncope. One year prior to his referral, he had been diagnosed with sick sinus syndrome (SSS) and received a cardiac pacemaker at our hospital, however, he suffered from a recurrence of syncope and was brought to our hospital by ambulance. After arriving, he lost consciousness and had convulsions in the emergency room, but recovered spontaneously. The results of physical examinations and a chest $\mathrm{X}$-ray were unremarkable. The ECG showed sinus rhythm with a slight PR prolongation of 0.26 sec., incomplete right bundle branch block (RBBB), and a bizarrely shaped ST segment elevation in $\mathrm{V}_{1-3}$ (Figure 1, top). However, he reported experiencing no chest pain. Two hours after his hospitalization, the patient suddenly developed polymorphic ventricular tachycardia followed by ventricular fibrillation (VF) (Figure 1, bottom). The delivery of an external DC shock successfully recovered sinus rhythm.

Laboratory findings, echocardiographic examination, and cardiac catheterization revealed no abnormality. We thus diagnosed as idiopathic VF (IVF). After the administration of prophylactic medical therapy, i.e., mexiletine and propranolol, VF did not recur. The ST elevation in the right precordial leads was gradually reduced after defibrillation, but it persisted.

Three years later, during a study-abroad program at the Good Samaritan Hospital, Los Angeles, California, I found an article by the Brugada brothers that surprised $\mathrm{me}^{\mathrm{l}}$. The Brugada brothers reported the eight patients of IVF with RBBB and persistent ST segment elevation, ${ }^{1)}$ whose ECG findings were very similar to those of the above-described patient.

Corresponding author: Masataka Sumiyoshi

Department of Cardiology, Juntendo University Nerima Hospital

3-1-10 Takanodai, Nerima-ku, Tokyo 177-8521, Japan.

TEL: +81-3-5923-3111 FAX: +81-3-5923-3217 E-mail: sumi@juntendo.ac.jp

[Received Jun. 28, 2021] [Accepted Jul. 13, 2021]

J-STAGE Advance published date: Oct. 11, 2021

Copyright (C) 2021 The Juntendo Medical Society. This is an open access article distributed under the terms of Creative Commons Attribution License (CC BY), which permits unrestricted use, distribution, and reproduction in any medium, provided the original source is properly credited. doi: 10.14789/jmj.JMJ21-0013-R 


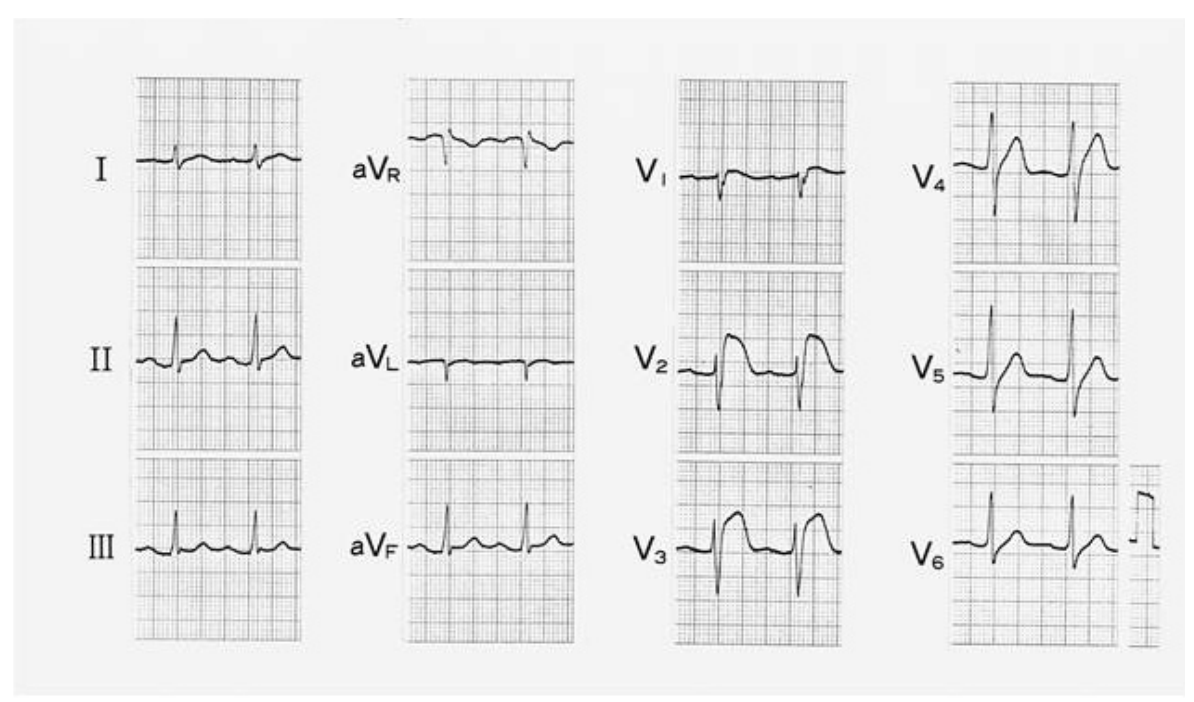

\section{4:57 PM}
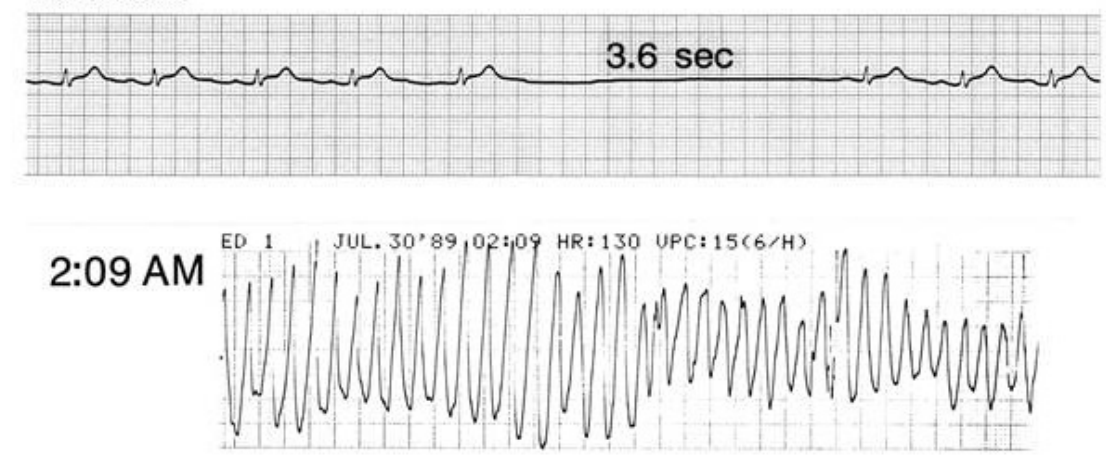

Figure 1 Top: Twelve-lead ECG just before ventricular fibrillation (VF) showed coved type ST elevation in leads $\mathrm{V}_{1-3}$. Middle: $\mathrm{ECG}$ monitoring revealed a sinus pause of $3.6 \mathrm{sec}$ in the evening. Bottom: Spontaneous VF occurred after midnight and was successfully converted to sinus rhythm by external DC shock. Cited with permission form ref.7.

Soon after returning to Japan, I wrote a case report about the patient I encountered at Juntendo University Hospital as I described before that was published in 1993 in Japanese Heart Journal. ${ }^{2)}$ I had searched for similar cases at related hospitals, but did not find any. This syndrome was later named "Brugada syndrome (BrS)". One day, Dr. Osamu Yamanaka, my senior by one year, asked me "Is there any relationship between $\mathrm{VF}$ and sinus node dysfunction?" At that time, I had not noticed the association of the two arrhythmias. Five years later (1998), the first genetic mutation of $\mathrm{BrS}$ was reported in Nature. ${ }^{3)}$ I was glad as our case report was cited in the report's references.

In 2003, I encountered another patient with BrS at Juntendo Shizuoka Hospital. The patient was a 62-year-old man who was referred for syncope. He had also experienced two episodes of nocturnal awakening with urinary incontinence. His ECG showed a typical Brugada-type ST elevation in the right precordial leads (Figure 2, left). During his hospitalization, ECG monitoring revealed asymptomatic episodes of sinus pause at midnight with a maximum duration of 6.6 seconds (Figure 2 , right). However, during the electrophysiologic study, the patient's sinus node function was normal and ventricular tachyarrhythmia could not be induced by the programmed electrical stimulation. On the basis of the multiple syncopal episodes including the nocturnal awakening with urinary incontinence and the typical ECG finding for BrS, the patient received with an implantable cardioverter-defibrillator (ICD), which successfully converted an episode of $\mathrm{VF}$ that occurred 2 months later (Figure 3). I remembered that the first case of $\mathrm{BrS}$ that I observed at Juntendo University Hospital had also suffered from sinus node dysfunction. I found several papers which revealed that a single muta- 


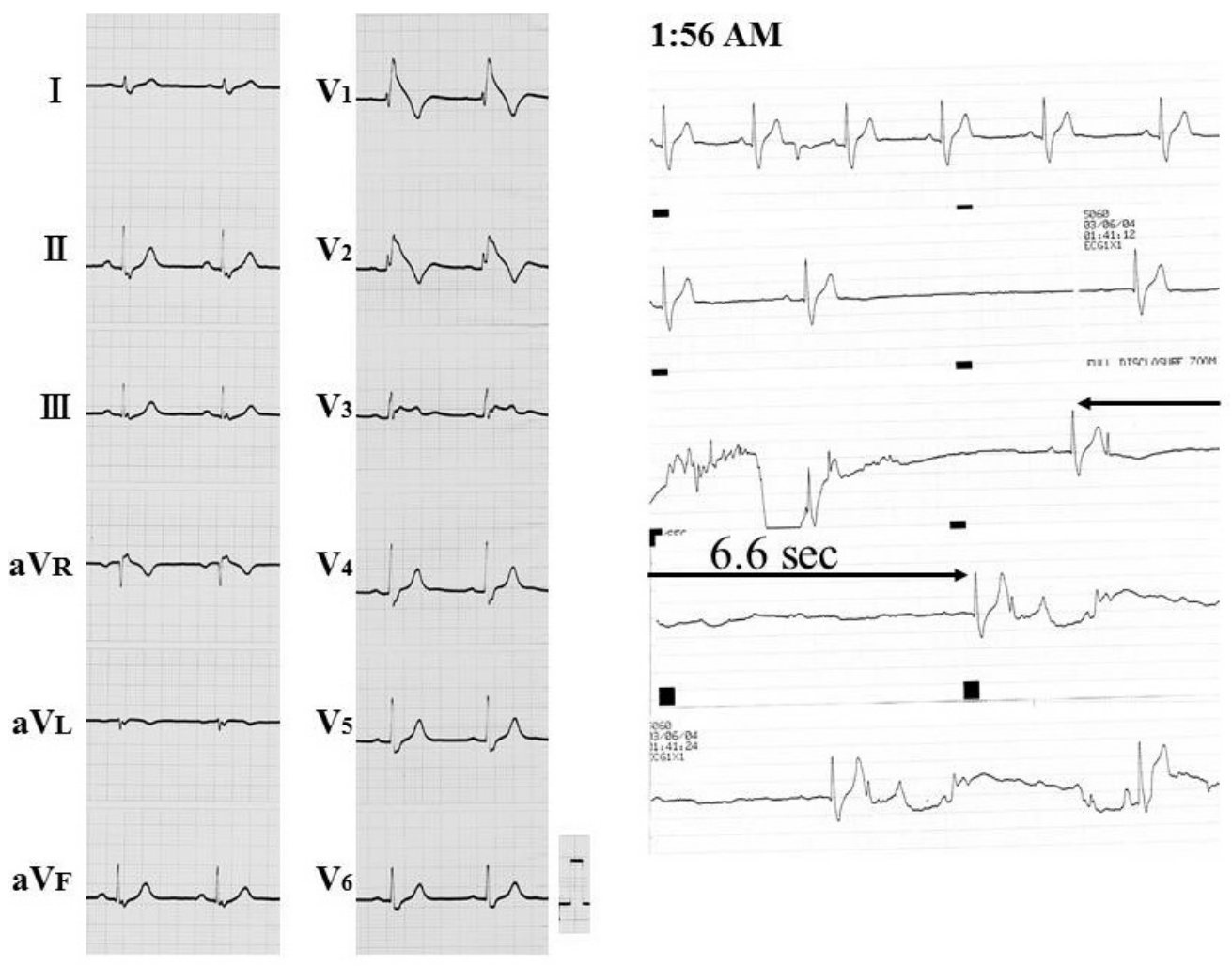

Figure 2 Left. ECG showed a typical Brugada-ECG pattern with coved-type ST elevation in $\mathrm{V}_{1}$ and $\mathrm{V}_{2}$. Right: ECG monitoring revealed asymptomatic sinus pauses with a maximum duration of $6.6 \mathrm{sec}$ after midnight during sleep. Cited with permission form ref.7.

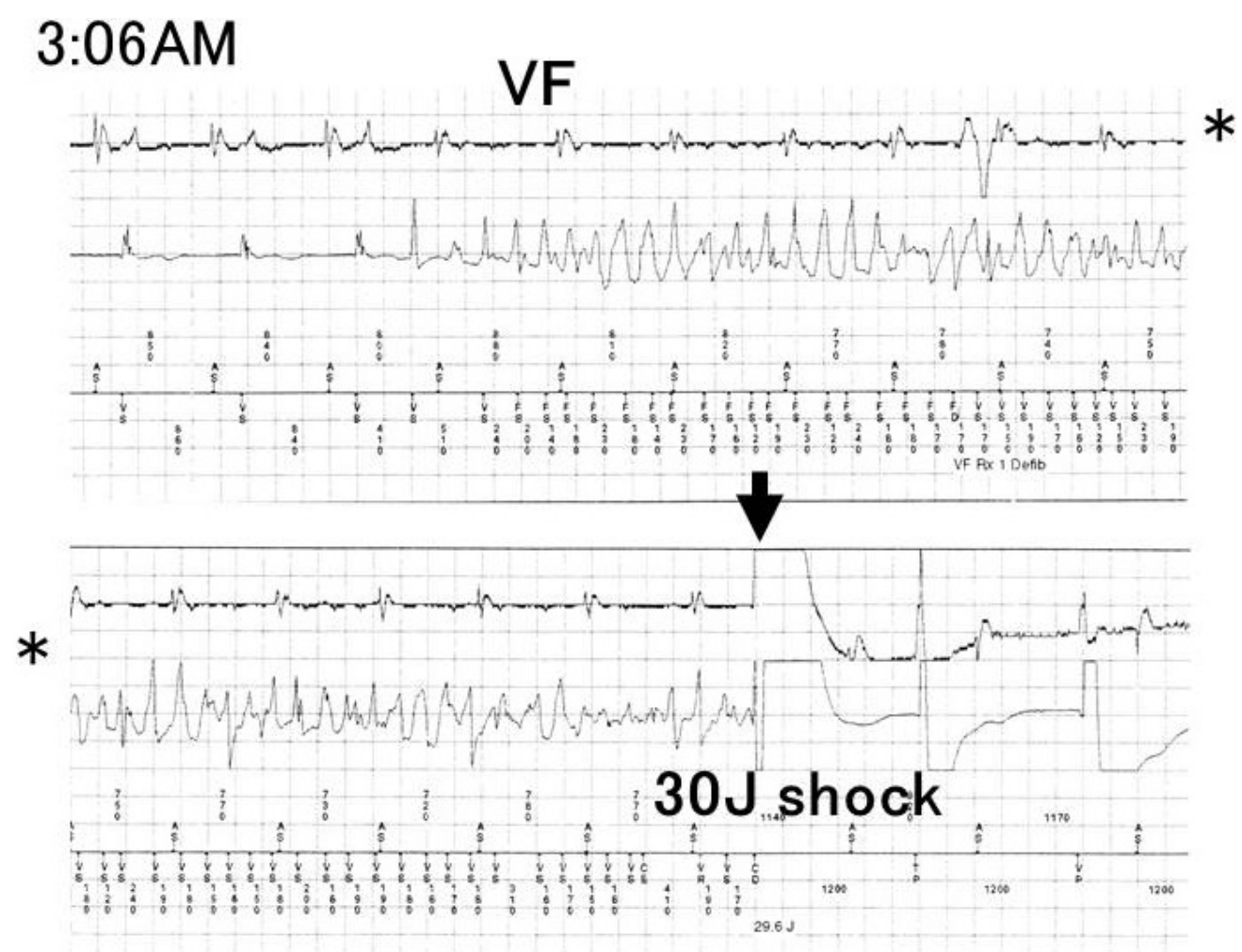

Figure 3 ECG from the implantable cardioverter-defibrillatior (ICD) showed successful conversion of an episode of spontaneous ventricular fibrillation to sinus rhythm at 3:06 a.m. Cited with permission form ref. 7 . 
tion in SCN5A could be a cause of both BrS and SSS. ${ }^{4-6)}$ At that point in time, we had only five patients diagnosed as having BrS at Juntendo University Hospital and affiliated hospitals, and three of the five patients coincidentally suffered from sinus node dysfunction. Then, I reported our three cases of $\mathrm{BrS}$ concomitant with sinus node dysfunction in 2005. ${ }^{\text {) }}$

At Juntendo University Hospitals, although the number of patients with BrS was very limited, there were many patients with SSS. We were able to study 487 consecutive patients implanted with a pacemaker for SSS at Juntendo University Hospital or Shizuoka Hospital during the period from 1971 to 2004. We retrospectively analyzed the patient's ECG results obtained just before the pacemaker implantations, and we identified a Brugada-type ECG in 14 patients (2.87\%) including four $(0.82 \%)$ with type 1 (the coved-type) and 10 (2.05\%) with type 2 (the saddleback-type). ${ }^{8)}$ Remarkably, two of the four patients with a type 1 ECG had experienced a spontaneous episode of VF. In 2010, we reported that the prevalence of typical Brugada-type (type 1) ECG among SSS patients seems to be higher compared to the general population: $0.82 \%$ in SSS patients and $0.05 \%-0.28 \%$ in adults of the general population. ${ }^{8)}$ The report's first author,

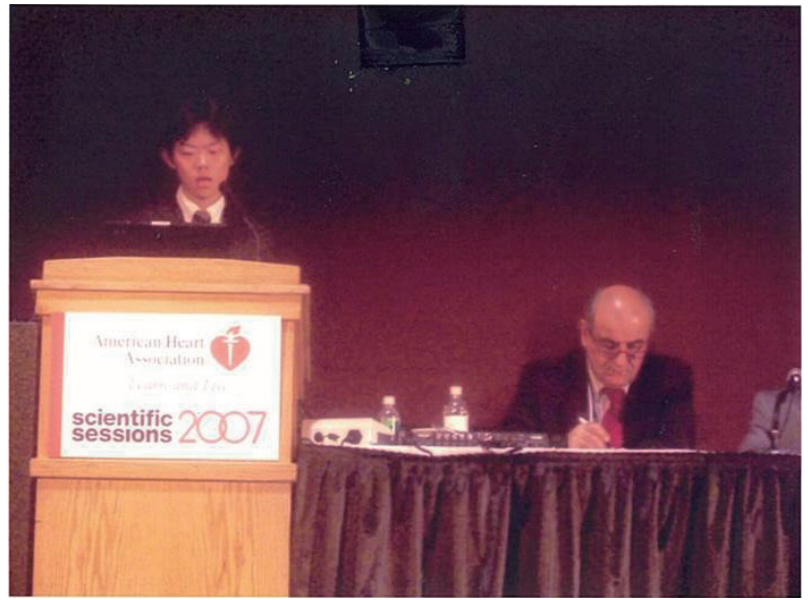

Figure 4 Dr. Hidemori Hayashi (left) making a presentation before Professor Joseph Brugada (right) at the AHA meeting in 2007.

Dr. Hidemori Hayashi, made a presentation about the report at the American Heart Association meeting in 2007 (Figure 4). Dr. Hayashi discussed his presentation with Professor Joseph Brugada (Figure 4, right), one of the Brugada brothers, who attended the meeting as a session chair-person. Dr. Hayashi won the Young Investigator's Award (YIA) from the Japanese College of Cardiology in 2007 for this study.

In 2018, we published the clinical review "Brugada

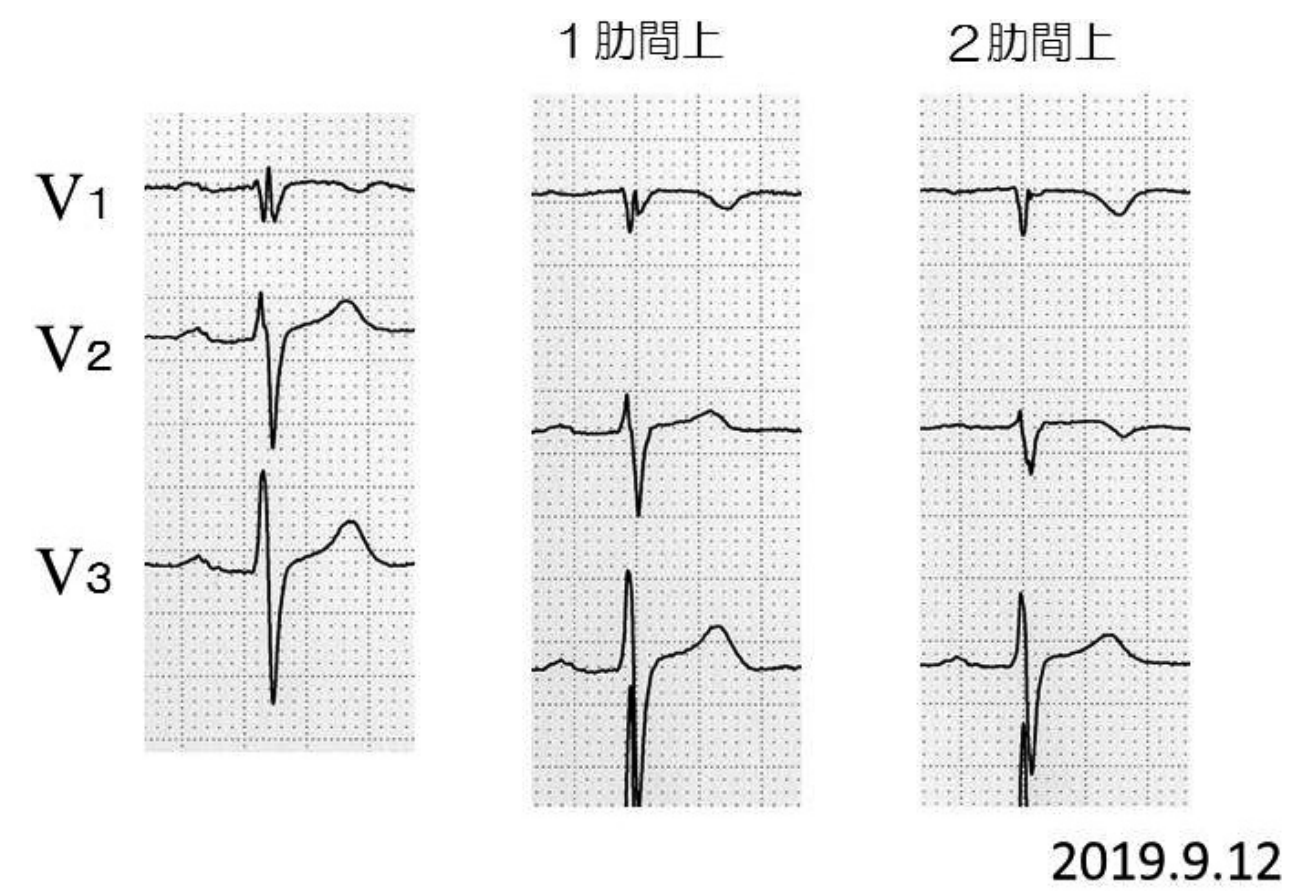

Figure 5 The ECG of the first patient with Brugada syndrome at Juntendo University Hospital, recorded 30 years after his last episode of ventricular fibrillation, no longer showing Brugada-type ECG findings. 
syndrome and sinus node dysfunction" in the Journal of Arrhythmia as a compilation of our studies concerning BrS and $\mathrm{SSS}^{9}$. My first BrS patient encountered during my night shift at Juntendo University Hospital had a documented episode of VF 32 years earlier, and he has been doing well without syncope or an ICD operation since then. His recent ECG, recorded 30 years after his last VF episode, no longer showed Brugada-type ECG findings (Figure 5).

\section{Epilogue}

I described a narrative concerning my clinical studies of BrS and SSS. These studies were inspired by a patient I encountered at Juntendo University Hospital. I believe that case report is very important for academic career because clinical studies may start with a clinical question in case presentation.

\section{Acknowledgements:}

I express my sincere gratitude to all the staff and members of the EP group at Juntendo University for their continuous support.

\section{Funding sources: None}

Disclosure: The author has no conflicts of interest to disclose.

\section{References}

1) Brugada P, Brugada J: Right bundle branch block, persistent ST segment elevation and sudden cardiac death: A distinct clinical and electrocardiographic syndrome. J Am Coll Cardiol, 1992; 20: 1391-1396.

2) Sumiyoshi M, Nakata Y, Hisaoka T, et al: A case of idiopathic ventricular fibrillation with incomplete right bundle branch block and persistent ST segment elevation. Jpn Heart J, 1993; 34: 661-666.

3) Chen Q, Kirsch GE, Zhang D, et al: Genetic basis and molecular mechanism for idiopathic ventricular fibrillation. Nature, 1998; 392: 293-296.

4) Smith JP, Koopmann TT, Wilders R, et al: A mutation in the human cardiac sodium channel (E161K) contributes to sick sinus syndrome, conduction disease, and Brugada syndrome in two families. J Moll Cell Cardiol, 2005; 38: 969-981.

5) Makiyama T, Akao M, Tsuji K, et al: High risk for bradyarrhythmic complications in patients with Brugada syndrome caused by SCN5A gene mutation. J Am Coll Cardiol, 2005; 46: 2100-2106.

6) Makita N, Behr E, Shimizu W, et al: The E1784K mutation in SCN5A is associated with mixed clinical phenotype of type 3 long QT syndrome. J Clin Invest, 2008; 118: 2219-2229.

7) Sumiyoshi M, Nakazato Y, Tokano T, et al: Sinus node dysfunction concomitant with Brugada syndrome. Circ J, 2008; 69: 946-950

8) Hayashi H, Sumiyoshi M, Yasuda M, et al: Prevalence of the Brugada-type electrocardiogram and incidence of Brugada syndrome in patients with sick sinus syndrome. Circ J, 2010; 74: 271-277.

9) Hayashi H, Sumiyoshi M, Nakazato $Y$, Daida H: Brugada syndrome and sinus node dysfunction. J Arrhythmia, 2018; 34: 216-221. 